\section{Daños en salud mental de cuidadores familiares de personas con Alzheimer}

\section{Mental health damages of family caregivers of people with Alzheimer's}

\author{
Edna Johanna Herrera Merchán ${ }^{1 *}$ \\ Elveny Laguado Jaimes ${ }^{2}$ \\ Lady Johana Pereira Moreno ${ }^{3}$ \\ 1. Psicóloga. Doctora en Neuropsicología Clínica. Especialista en Salud Ocupacional. Docente- \\ Investigadora. Facultad de Investigaciones, Universidad Manuela Beltrán. Facultad, \\ sede Bucaramanga. Facultad de Psicología, Universidad Cooperativa de Colombia, sede \\ Bucaramanga. Colombia \\ 2. Enfermera. Magíster en Enfermería. Especialista en Salud Ocupacional. Especialista en \\ Docencia Universitaria. Profesora-Investigadora, Facultad de Enfermería. Universidad \\ Cooperativa de Colombia, sede Bucaramanga. Colombia. \\ 3. Psicóloga. Magíster en Neuropsicología y Educación. Especialista en Docencia Universitaria. \\ Especialista en Gerencia de la Salud Ocupacional. Profesora Investigadora, Facultad de \\ Psicología. Universidad Cooperativa de Colombia, sede Bucaramanga. Colombia.
}

*Autor para correspondencia.

Correo electrónico: ejohannaherrera@gmail.com (Edna Johanna Herrera).

Recibido el 14 de noviembre de 2018; aceptado el 11 de diciembre de 2018

\section{RESUMEN}

Objetivo: Valorar daños en salud mental de cuidadores familiares de personas con demencia de institución psiquiátrica para proponer acciones y mitigar sus efectos. Método: estudio descriptivocorrelacional de corte transversal en 28 sujetos con cuestionarios de salud SF-36, escala de sobrecarga de Zarit, cuestionario del paciente PHQ-9, análisis de porcentaje y pruebas de correlación t-Student y Pearson. Resultados: Género predominante, mujeres (85,2\%); edad media, 59,3 años; presentaron depresión menor un 44,4\% y mayor un $14,8 \%$, sobrecarga ligera un $22,2 \%$ y sobrecarga intensa un $37,0 \%$, correlación negativa significativa $(p=0,02)$ entre depresión y vitalidad.

La calidad de vida a nivel físico en un cuidador de 55 años o más se encuentra disminuida (media $=23,3$ ). Conclusiones: De acuerdo con los resultados, indican para este estudio que factores como tener más de 55 años, percibir una peor función física y sentirse menos vital son algunas variables asociadas a la aparición de depresión y sobrecarga.

PALABRAS CLAVE: Cuidadores familiares, Alzheimer, depresión, sobrecarga, calidad de vida, demencia.

\section{ABSTRACT}

Objective: to evaluate mental health damages of family caregivers of people with dementia of a psychiatric institution to propose actions and mitigate their effects. Method: descriptive-correlational crosssectional study in 28 subjects with SF-36 health questionnaires, Zarit overload scale, PHQ-9 patient questionnaire, percentage analysis and t-Student and Pearson correlation tests. Results: predominant gender women $(85.2 \%)$; mean age of 59.3 years, had a minor depression of $44.4 \%$ and greater by $14.8 \%$, slight overload in $22.2 \%$ and intense overload in $37.0 \%$, significant negative correlation $(p=$ 0.02 ) between depression and vitality. The quality of life at a physical level in a caregiver of 55 years or more is diminished ( mean $=23.3$ ). Conclusions: According to the results indicate for this study that factors such as being over 55 years of age, perceiving a worse physical function, and feeling less vital are some variables associated with the appearance of depression and overload.

KEYWORDS: Family Caregivers, Alzheimer's, Depression, Overload, Quality of Life, Dementia.

\section{- INTRODUCCIÓN}

Las enfermedades crónico-degenerativas como el Alzheimer incrementan el grado de dependencia del paciente, definido como un estado permanente de pérdida de la autonomía física, mental, intelectual y sensorial, que precisan ayudas importantes para realizar actividades básicas de la vida diaria, además de cuidados de manera continuada (ocupando gran parte del tiempo del cuidador) y prolongada en el tiempo ${ }^{1-3}$; de igual forma esto afecta significativamente al estilo de vida del cuidador, pues debe pensar en forma diferente y tomar decisiones en medio de alternativas complicadas ${ }^{4,5}$.

Por lo anterior surgen cambios que alteran la dinámica familiar, puesto que son estos quienes tendrán la demanda de cuidar a una persona con demencia. Es aquí donde aparece el cuidador familiar, que sin tener ninguna formación o habilidad realizará acciones de cuidado ${ }^{4}$, que afectarán seria- mente a su salud física y mental, aspecto estudiado por varios autores ${ }^{6}$. En un metanálisis de 84 artículos reportan que las personas cuidadoras presentan daños en la salud mental en variables como depresión, estrés, autoeficacia y bienestar subjetivo en comparación con personas no cuidadoras; concluyen que el acto de cuidar afecta gravemente a la salud física y mental, dejando vulnerable al cuidador ante cualquier enfermedad. Es por ello por lo que en muchos países industrializados se adoptan políticas de apoyo económico hacia cuidadores de personas discapacitadas ${ }^{7}$.

Otros estudios revelan los efectos de la depresión en cuidadores para las variables de calidad de vida y sobrecarga, generando daños en la función física como aumento del dolor y problemas para realizar actividades físicas $^{8}$. En el rol emocional disminuye la capacidad de afrontamiento emocional, también la vitalidad en variables como sentirse enfermo ahora más que antes, y en la función social al disminuir actividades sociales del cuidador como son las visitas a familiares y amigos o reuniones sociales? 
De acuerdo con lo anterior, la calidad de vida tiene un impacto en la díada cuidador-paciente ${ }^{10,11}$, factores asociados a la cronicidad de la enfermedad, deterioros cognitivos, funcionales y cambios comportamentales del paciente que incrementan la dependencia hacia su cuidador ${ }^{12}$, además de otros factores como la renta disponible del hogar, el nivel de actividad laboral del cuidador, los servicios formales para los cuidados y variables relativos a las características de la unidad familiar ${ }^{17}$, además de relaciones negativas y positivas debidas al cuidado del paciente que incrementan dificultades en el manejo de la enfermedad ${ }^{13}$.

\section{- OBJETIVO}

Valorar daños en salud mental de cuidadores familiares de personas con demencia de la Institución Psiquiátrica ISNOR en el segundo semestre de 2017. Información relevante para implementar acciones que mitiguen los efectos del cuidado.

\section{- METODOLOGÍA Y MATERIALES}

El tipo de estudio es descriptivo-correlacional de corte transversal con 28 cuidadores, 4 hombres y 24 mujeres, seleccionados mediante muestreo por conveniencia y que cumplieron los criterios de inclusión tales como ser cuidadores de familiares con enfermedad de Alzheimer, nivel de escolaridad básico, ser alfabetos, no estar diagnosticados con enfermedad psiquiátrica que pudiera alterar los resultados de las pruebas psicológicas, y cuidar a su familiar durante más de 3 meses, por un período aproximado de cinco o más horas diarias. El contacto con los cuidadores mediante la base de datos autorizada por el Instituto del Sistema del Nervioso del Oriente (ISNOR) de Bucaramanga, previo consentimiento informado de los participantes, en el cual se protegen los datos mediante convenio 1 de febrero del 2017; los instrumentos previo consentimiento informado según Declaración de Helsinki, sin que la investigación generara ningún daño a la salud ni mental ni física de los participantes. Se aplicó a cada participante el cuestionario de salud Health Survey (SF-36) $)^{14}$, la escala de sobrecarga del cuidador de Zarit ${ }^{15}$, el cuestionario sobre la salud del paciente (Patient Health Questionnaire [PHQ-9]) ${ }^{16}$ y un cuestionario de datos sociodemográficos. El estudio fue aprobado por un Comité de Bioética de la Universidad Cooperativa de Colombia. En el análisis de datos se realizó una descripción de frecuencias en porcentajes para variables sociodemográficas y de salud mental, y correlación estadística mediante pruebas $t$ de Student y correlación de Pearson.

\section{- RESULTADOS}

Fueron valorados 28 cuidadores familiares, con una edad promedio de 59,3 ańos, en un rango de edad de 35 hasta 80 ańos; más de la mitad de los cuidadores $(56,0 \%)$ tenía 55 años o más. Mayor proporción de mujeres $(85,2 \%)$ en comparación con los hombres (14,3\%); sus profesiones eran amas de casa $(32,0 \%)$, en menor proporción pensionadas $(28,0 \%)$ y trabajador independiente $(20,0 \%)$. El parentesco con el paciente era, en algo más de la mitad de los cuidadores, ser hija del paciente $(52,6 \%)$, en menor proporción, esposa $(15,8 \%)$; cabe resaltar que todos los cuidadores presentan algún vínculo familiar con el paciente.

En la tabla 1 se muestran los resultados del PHQ9 donde un 59,2\% de los cuidadores presenta algún trastorno depresivo, de los cuales un $44,4 \%$ tiene un cuadro depresivo menor y un $14,8 \%$ un cuadro depresivo mayor.
Tabla 1. Cuestionario sobre la salud mental del cuidador (PHO-9)

\begin{tabular}{|l|c|c|c|}
\hline $\begin{array}{l}\text { Puntuación } \\
\text { PH0-9 }\end{array}$ & $\begin{array}{c}\text { Categorías } \\
\text { PHO-9 }\end{array}$ & Frecuencia & Porcentaje \\
\hline$\leq 4$ & Sin depresión & 11 & 40,70 \\
\hline $5-14$ & Depresión leve & 12 & 44,40 \\
\hline$\geq 15$ & Depresión mayor & 4 & 14,80 \\
\hline Total & & $\mathbf{2 7}$ & $\mathbf{1 0 0 , 0}$ \\
\hline
\end{tabular}

Fuente: base de datos del grupo de investigadores.

De acuerdo con la prueba de $\chi^{2}$, existe una asociación estadísticamente significativa entre pertenecer a uno $\mathrm{u}$ otro rango de edad y presentar algún trastorno depresivo $\left(\chi^{2} 7,865\right.$, sig $\left.=0,020\right)$, teniendo en cuenta que en este estudio predomina la edad de mayores de 55 años.

Mediante la escala de sobrecarga de Zarit, los resultados de la investigación en la tabla 2 destacan que un poco más de la mitad de los cuidadores $(59,2 \%)$ presenta algún nivel de sobrecarga, de los cuales un $22,2 \%$ presenta un nivel de sobrecarga ligera y un $37,0 \%$ presenta un nivel de sobrecarga intensa.

Tabla 2. Escala de sobrecarga del cuidador (Zarit)

\begin{tabular}{|l|c|c|c|}
\hline $\begin{array}{l}\text { Puntuaciones } \\
\text { Zarit }\end{array}$ & $\begin{array}{c}\text { Categorías } \\
\text { Zarit }\end{array}$ & Frecuencia & Porcentaje \\
\hline$<46$ & No sobrecarga & 11 & 40,7 \\
\hline $46-55$ & Sobrecarga leve & 6 & 22,2 \\
\hline$>55$ & $\begin{array}{c}\text { Sobrecarga } \\
\text { intensa }\end{array}$ & 10 & 37,0 \\
\hline Total & & $\mathbf{2 7}$ & $\mathbf{1 0 0 , 0}$ \\
\hline
\end{tabular}

Fuente: base de datos del grupo de investigadores.

La edad para determinar la sobrecarga del cuidador (Zarit), en la prueba de $\chi^{2}$, tiene una asociación estadísticamente significativa entre pertenecer a uno u otro rango de edad y presentar algún nivel de sobrecarga, donde a mayor edad mayor sobrecarga $\left(\chi^{2} 6,663\right.$, sig $\left.=0,036\right)$.

El cuestionario de salud SF-36 identificó estados positivos y negativos de salud de los cuidadores, así como su salud física y mental. Los resultados dejan ver 8 dimensiones del estado de salud del cuidador: función física, rol físico, dolor corporal, salud general, vitalidad, función social, rol emocional y salud mental. Los reportes referencian mejoría de salud ahora que hace un año $(33,3 \%)$, y peor salud ahora que hace un año $(33,3 \%)$. Al relacionar depresión del cuidador (PHQ-9) con el nivel de vitalidad (SF-36), se pudo identificar una correlación negativa y estadísticamente significativa (sig.= 0,002$)$; esto es que, a mayor depresión del cuidador, su vitalidad es menor (tabla 3).

Tabla 3. Correlaciones para depresión en cuidador (PHO-9) y su vitalidad (SF-36)

\begin{tabular}{|c|c|c|c|}
\hline & & & Vitalidad \\
\hline PHO-9 & $\begin{array}{c}\text { Correlación de } \\
\text { Pearson }\end{array}$ & & $-0,570$ \\
\hline & Sig. (bilateral) & & 0,002 \\
\hline & N & 27 & 27 \\
\hline
\end{tabular}

Fuente: base de datos del grupo de investigadores. 


\section{- DISCUSIÓN}

La investigación encontró que los cuidadores de este estudio muestran un trastorno depresivo de un 59,2\%, comparado con otro estudio en Cali, Colombia, donde reportan mayores índices de depresión en cuidadores familiares en comparación con una muestra control, lo que sugiere la necesidad de tener servicios en salud mental y otros servicios del cuidado ${ }^{17}$.

Culturalmente, en Colombia se tiende a cuidar a los familiares más que a internarlos en centros geriátricos. Los cuidadores participantes en el estudio convivían con la persona con demencia, predominio de género femenino, dedicación como amas de casa 32,0\%; datos que son similares al estudio en Colombia, con un $81,2 \%$ de cuidadores de género femenino, un 41,6\% dedicados al hogar ${ }^{18}$.

En otra investigación se encontró que las características asociadas a altas tasas de depresión fueron la edad avanzada, el género femenino más frecuente que el masculino, vivir con el paciente, y la relación familiar (ser hija o cónyuge), además de prestar cuidados y tener peor función física ${ }^{19}$.

Dato relacionado también con este estudio puesto que se encontró que más de la mitad de los cuidadores son mujeres, seguido por un parentesco de ser hija, percibir menos vitalidad y ser mayor de 55 ańos. Con esto se puede evidenciar que los factores mencionados en otras investigaciones se relacionan con los datos obtenidos en este estudio ${ }^{20}$.

Otro factor que influye en la aparición de la depresión son los problemas de comunicación con el paciente; se definen predictores de la depresión en cuidadores dados por la dependencia en las actividades instrumentales de la vida diaria, y los problemas de la comunicación con el paciente que produce altos niveles de insatisfacción en el cuidador, quien a menudo no comprende las necesidades del paciente lo que genera depresión ${ }^{21}$. En este estudio no se analizaron variables de comunicación en la díada cuidador-paciente; sería un factor a tener en cuenta para analizar la depresión en cuidadores familiares.

\section{- CONCLUSIONES}

Los cuidadores familiares presentaron problemas de depresión y sobrecarga, disminución de calidad de vida en función física, cuidadores mayores de 55 ańos tienen impedimentos para el cuidado por requerir esfuerzo físico, y mayores niveles de vitalidad; además, a mayor edad de los cuidadores, mayor sobrecarga. Los cuidadores con depresión muestran disminuida en forma significativa su energía vital y la capacidad física en su cotidianidad.

La depresión es un factor que se presentó en este estudio y en otros de Colombia. Por tales motivos es indispensable que los cuidadores familiares de personas con demencia puedan tener asistencia en salud mental y otros recursos para ejercer labores del cuidado

\section{Agradecimientos}

Las autoras desean expresar su gratitud al Comité Nacional para el Desarrollo de la Investigación (CONADI) de la Universidad Cooperativa de Colombia que financió el proyecto de investigación; también extender el agradecimiento a las estudiantes que pertenecían a las facultades de Enfermería y Psicología, quienes apoyaron esta investigación en la obtención de los datos, y al Instituto del Sistema Nervioso del Oriente (ISNOR).

\section{Conflicto de intereses}

Las autoras declaran no tener ningún conflicto de intereses relacionado con este artículo.

\section{- BIBLIOGRAFÍA}

1. Flores N, Jenaro C. Moro L, Tomșa R. Salud y calidad de vida de cuidadores familiares y profesionales de personas mayores dependientes: estudio comparativo. Eur J Invest Health Psychol Educ [Internet]. 2014 [fecha de consulta 22 de mayo 2018]:4(2):79-88. Disponible en: https://formacionasunivep.com/ejihpe/index.php/ journal/article/view/73

2. Sánchez RT, Molina EM, Gómez-Ortega OR. Intervenciones de enfermería para disminuir la sobrecarga en cuidadores: un estudio piloto. Rev Cui. 2016;7(1):1171-84

3. Cerquera A, Galvis M. Efectos de cuidar personas con Alzheimer: un estudio sobre cuidadores formales e informales. Pensamiento psicológico. 2014;12(1):149-67.

4. Hernández NE, Moreno CM, Barragán JA. Necesidades de cuidado de la díada cuidador-persona: expectativa de cambio en intervenciones de enfermería. Rev Cuid. 2014 (consulta 22 de mayo de 2018);5(2):748-56. Disponible en: https://revistacuidarte.udes.edu. co/index.php/cuidarte/article/view/87/247

5. Delgado E, Suárez O, Dios del Valle R, Valdespino I, Sousa Y Braña G. Características y factores relacionados con sobrecarga en una muestra de cuidadores principales de pacientes ancianos con demencia. Semergen. 2014;40(2):57-64.

6. Timonet-Andreu E, Morales J M, Canca JC, Rivas-Ruíz F, Mesa R, Sepúlveda J. et al. Calidad de vida relacionada con la salud y sobrecarga de cuidadores de pacientes ingresados con insuficiencia cardiaca. Enferm Cardiol [Internet]. 2013 lacceso 23 de mavo de 2018);(58):50-5. Disponible en: https://www.enfermeriaencardiologia.com/wp-content/uploads/58_59_07.pdf

7. Pinquart M. Sörensen S. Differences Between Caregivers and Non Caregivers in Psychological Health and Physical Health: A Meta Analysis. Psychol Aging [Internet]. 2003 (acceso 22 de mayo de 2018);18(2):250-67. Disponible en: https://www.ncbi.nlm.nih.gov/ pubmed/12825775
8. Mora-Castañeda B, Márquez-González M, Fernández-Liria A de la Espriella R, Torres N, Arenas A. Variables demográficas y clínicas relacionadas con la carga y el afrontamiento de los cuidadores de personas diagnosticadas de esquizofrenia. Rev Colomb Psiquiat [Internet] 2016 (acceso 18 de mayo de 2018):47(1):13-20. Disponible en: https:/ reader.elsevier.com/reader/sd/pii/S0034745016301184?token=81D7612539DF211FE1700F6EAD50767D9CBFE827F4199064BA2CC 326DD01BF4305AC1ADF17CCD786654C4BA94E5D5412

9. Carreño Moreno SP, Chaparro Díaz L. Reconstruyendo el significado de calidad de vida de los cuidadores en el cuidado: una meta síntesis. Av. enferm. [Internet]. 2015 [acceso 18 de abril de 20181;33(1):55-66. Disponible en: https://revistas.unal.edu.co/index.php/avenferm/article/view/48103/60874

10. Deví Bastida J, Naqui Esteve M, Jofre Font S, Fetscher Eickhoff A. Relación entre el burnout y la calidad de vida del cuidador de personas con demencia. Gerokomos; 2016 [acceso 15 de mayo de 2018);27(1):19-24. Disponible en: http://scielo.isciii.es/pdf/geroko/v27n1/05_revision_04.pdf

11. Ávila J, Vergara M. Calidad de vida en cuidadores informales de personas con enfermedades crónicas. Aquichan. 2014 [acceso 18 de mayo de 2018);14(3):417-29. Disponible en: http://www.redalyc org/articulo.oa? id=74132361011

12. Perdomo-Romero AY, Ramírez-Perdomo CA. Perception of quality of life in caregivers of patients with dementia. Rev Cient Soc Esp Enferm Neurol (English ed.). 2017;46:26-31.

13. Sapin M, Widmer ED, Iglesias K. From support to overload: Patterns of positive and negative family relationships of adults with mental illness over time. Social Networks. 2016 October lacceso 22 de mayo de 2018];47:59-72. Disponible en: https://www.sciencedirect. com/science/article/pii/S0378873316301381

14. Ware JE, Kosinski MA, Keller SD. SF-36 Physical and Mental Health Summary Scales: A User's Manual. Boston, MA: Health Assessment Lab; 1994
15. Bianchi M, Flesch LD, Valeska da Costa Alves E, Sathler Taveres Batistoni S, Liberalesso Neri A. Indicadores psicométricos de la Zarit Burden Interview aplican en ancianos cuidadores de otros ancianos. Rev. Latino-Am. Enfermagem [Internet]. 2016 [citado 28 de noviembre de 2018];24:e2835. Disponible en: http://www.scielo.br/scielo. php?script=sci_arttext\&pid=S0104-11692016000100433\&lng=en Epub Nov 28, 2016. http://dx.doi.org/10.1590/1518-8345.1379.2835

16. Spitzer R, Kroenke K. Williams J. Validation and utility of a self-report Version of PRIME-MD: the PHQ Primary Care Study. J Am Med Assoc. 1999 [acceso 20 de junio de 2016);282(18):1737-44. Disponible en: https://www.ncbi.nlm.nih.gov/pubmed/10568646

17. Posner B, Sutter M, Perrin PB, Hoyos GR, Buraye JA, Arango-LaspriIla JC. Estudio sobre la salud mental y la calidad de vida de un grupo de cuidadores de personas con demencia y un grupo control en Cali, Colombia. Psicología desde el Caribe. 2014:32(1):1-26.

18. Cerquera Córdoba AM, Pabón Poches DK. Resiliencia y Variables Asociadas en Cuidadores Informales de Pacientes con Alzheimer Revista Colombiana de Psicología. 2016:25(1):33-46.

19. De Fazio P, Ciambrone P, Cerminara G, Barbuto E, Bruni A, Gentile P. et al. Sintomas depresivos en cuidadores de pacientes con demencia: variables demográficas y carga. Intervenciones clínicas en el envejecimiento. 2015:10:1085.

20. Givens JL, Mezzacappa C, Heeren T, Yaffe K, Fredman L. Depressive symptoms among dementia caregivers: role of mediating factors. Am J Geriatr Psychiatry. 2014 [acceso 20 de agosto de 2018);22(5):481-8. Disponible en: https://www.ncbi.nlm.nih.gov/ pubmed/23567432

21. Wong PK, Cheung G, Fung R, Koo S, Sit E, Pun SH, et al. Patien and Caregiver Characteristics Associated with Depression in Dementia Caregivers. J Psychol Chinese Soc. 2008;9(2):195-224.Disponible en: https://cup.cuhk.edu.hk/chinesepress/journal/JPCS9.2/ JPCS9.2_195-224.pdf 